\title{
Multi-GHz Burst-Mode Fiber Lasers
}

\author{
F. Ömer Ilday ${ }^{1,2,3}$ \\ ${ }^{1}$ UNAM - National Nanotechnology Research Center, Bilkent University, Ankara, Turkey \\ ${ }^{2}$ Department of Physics, Bilkent University, Ankara, Turkey \\ ${ }^{3}$ Department of Electrical and Electronics Engineering, Bilkent University, Ankara, Turkey
}

\begin{abstract}
Ultrafast lasers with $\mathrm{GHz}$ repetition rates is necessary to access the highly efficient ablation-cooled lasermaterial processing regime. Burst-mode operation allows access to such high repetition rates at microjoule-level pulse energies without needing $\mathrm{kW}$-level average powers. This talk will review the development of the first femtosecond burst-mode fiber lasers to date.
\end{abstract}

Keywords- ultrafast lasers, fiber lasers, ultrafast micromachining, laser-material interactions

Ultrafast lasers allow thermal damage-free ablation irrespective of the material type. However, this is a slow and inefficient process, because enough time has to pass between subsequent pulses for the material to cool down to avoid heat buildup. We recently demonstrated ablation-cooled lasermaterial, whereby ultrafast pulses are sent so quickly one after average power of the laser system can be lowered indefinitely. The first burst-mode lasers employed pulse pumping of the amplifier just before the arrival of each burst to avoid amplified spontaneous emission between the bursts. This way, burst repetition rates of $1-50 \mathrm{kHz}$ or burst-on-demand operation was possible. Following developments that made the generation of high average powers much more accessible, we have moved on to burst repetition rates that exceed $100 \mathrm{kHz}$, for which pulsed pumping is not necessary, thus significantly simplifying the laser design.

This talk will review the development of burst-mode fiber lasers [3], as well as the highly efficient regime of ablationcooled material processing, enabled these lasers. In the future, radically different and simpler laser designs appear to be possible, designs that do not use the MOPA architecture.

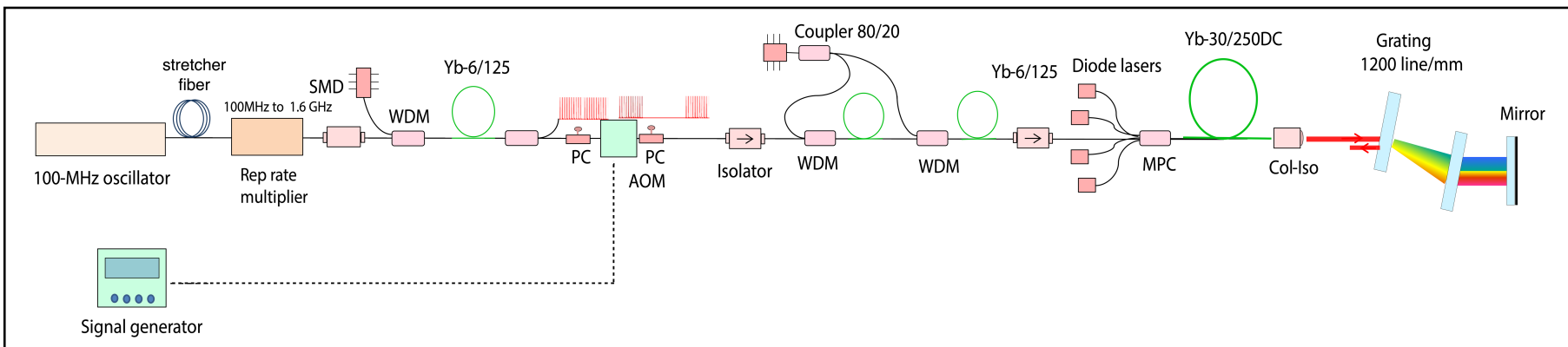

Fig. 1. Typical setup schematics of one of the earlier burst-mode lasers. This particular laser generated 250 -fs pulses at $1.6 \mathrm{GHz}$ with $50 \mathrm{~W}$ of average power.

another (within 100's picoseconds of each other) that there is insufficient time for heat to diffuse away from the processing region $[1,2]$. In this regime, the interaction of the pulses with the target material is no longer isolated, but rather, many thousands of pulses interact collectively with the material. Further, the ablation rate increases by orders of magnitude, and consequently, the ablation process becomes the dominant heat removal mechanism, and the rest of the target material remains cool and without damage.

Access to the ablation-cooled regime requires $\mathrm{GHz}$ repetition rates, which would normally require $\mathrm{kW}$ levels of average power for a modest pulse energy of $1 \mu \mathrm{J}$. In response to this challenge, circa 2011, we started developing the first fiber laser-amplifiers that implemented the commonly used masteroscillator power-amplifier (MOPA) architecture. Still, these MOPAs operated in the so-called burst-mode operation. In this mode, the laser produces a temporally closely spaced group of pulses, a burst, which is then repeated at a relatively low rate. This way, GHz-level repetition rates can be achieved within the burst. By keeping the burst repetition rate low, the overall

\section{ACKNOWLEDGMENT}

This work was supported partially by the European Research Council (ERC) Consolidator Grant ERC-617521 NLL.

\section{REFERENCES}

[1] C. Kerse, H. Kalaycıŏglu, P. Elahi, B. Çetin, D. K. Kesim, Ö. Akçaalan, S. Yavaş, M. D. Aşık, B. Öktem, H. Hoogland, R. Holzwarth, and, F. Ö. Ilday, "Ablation-cooled material removal with ultrafast bursts of pulses," Nature 537, 84-88 (2016).

[2] P. Elahi, H. Kalaycioglu, Ö. Akçaalan, C. Ertek, K. Eken, F. Ö. Ilday, "High-power Yb-based all-fiber laser delivering 300-fs pulses for highspeed ablation-cooled material removal," Opt. Lett. 43, 535-538 (2018).

[3] H. Kalaycioglu, P. Elahi, F. Ö. Ilday, Ö. Akcaalan, "High-repetition-rate ultrafast fiber lasers for material processing," IEEE J. Sel. Topics Quantum Electron. 24, 8800312 (2018). 